\title{
Correction to: The starch-binding domain as a tool for recombinant protein purification
}

\author{
D. Guillén ${ }^{1,2} \cdot$ S. Moreno-Mendieta ${ }^{3} \cdot$ P. Aguilera ${ }^{1} \cdot$ S. Sánchez ${ }^{1} \cdot$ A. Farres $^{4} \cdot$ R. Rodríguez-Sanoja $^{1}$
}

Published online: 18 September 2021

๑) Springer-Verlag GmbH Germany, part of Springer Nature 2021

\section{Correction to: Appl Microbiol Biotechnol (2013) 97:4141-4148 https://doi.org/10.1007/s00253-013-4778-0}

The original version of this article, unfortunately, contains mistakes introduced during the publishing process.
Panels for AcrSBDtag and CRSBDtag in Figure 3 are identical. In the submitted manuscript, the figure was correct. The correct figure is given below:

The original article can be found online at https://doi.org/10.1007/ s00253-013-4778-0.

R. Rodríguez-Sanoja

romina@biomedicas.unam.mx

1 Departamento de Biología Molecular y

BiotecnologíaInstituto de Investigaciones Biomédicas,

Universidad Nacional Autónoma de México, México,

Distrito Federal 04510, México

2 Programa de Posgrado en Ciencias Bioquímicas,

Universidad Nacional Autónoma de México, México,

Distrito Federal 04510, México

3 Programa de Doctorado en Ciencias Biomédicas, Universidad Nacional Autónoma de México, México, Distrito Federal 04510, México

4 Departamento de Alimentos y Biotecnología Facultad de Química, Universidad Nacional Autónoma de México, México, Distrito Federal 04510, México 


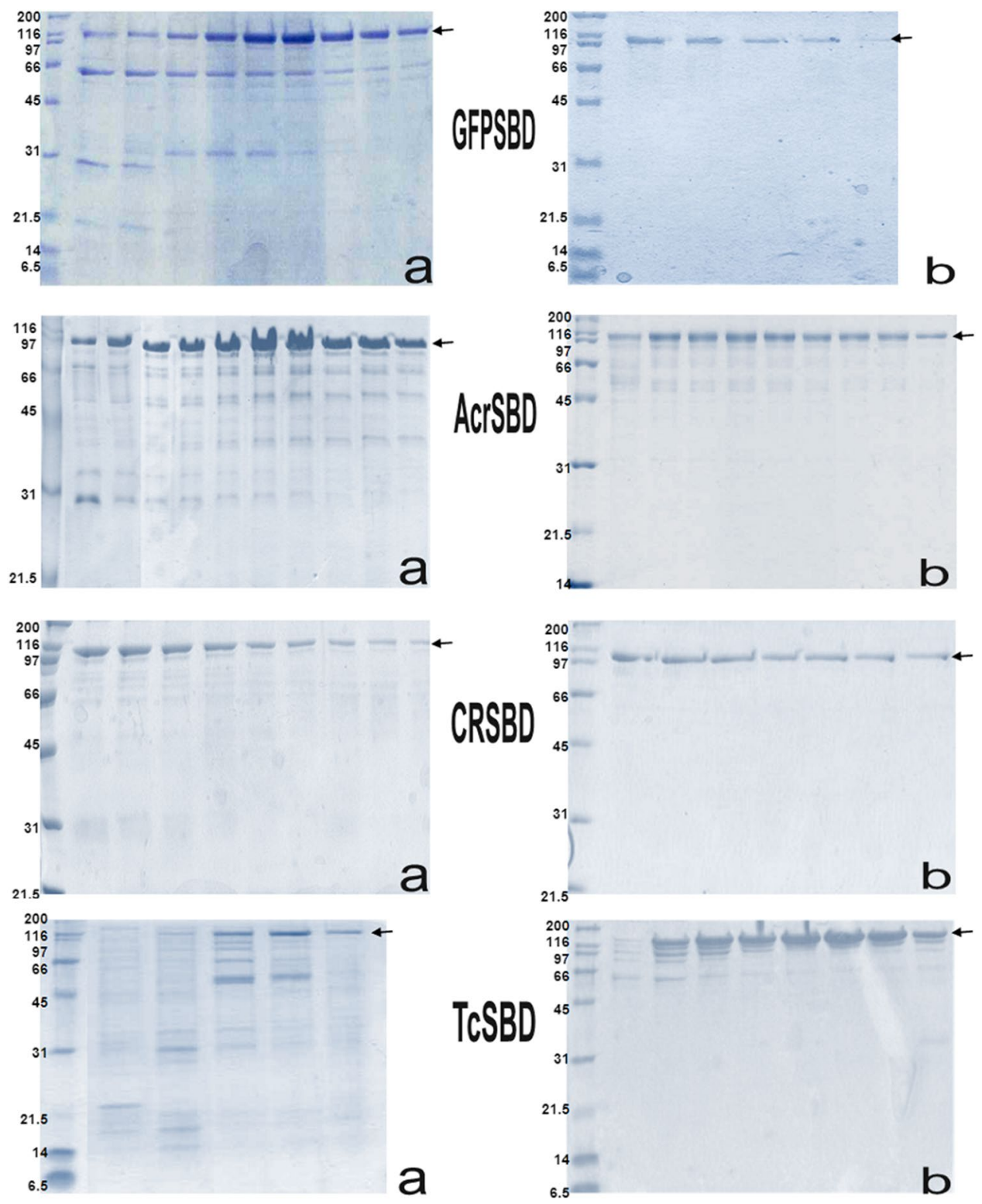

Publisher's note Springer Nature remains neutral with regard to jurisdictional claims in published maps and institutional affiliations. 